\title{
GRB 050223: a dark GRB in a dusty starburst galaxy ${ }^{\star}$
}

\author{
L. J. Pellizza ${ }^{1,2}$, P.-A. Duc ${ }^{1}$, E. Le Floc'h ${ }^{3,4}$, I. F. Mirabel ${ }^{5}$, L. A. Antonelli' ${ }^{6}$, S. Campana ${ }^{7}$, G. Chincarini ${ }^{7,8}$, \\ A. Cimatti ${ }^{9}$, S. Covino ${ }^{7}$, M. Della Valle ${ }^{9}$, F. Fiore ${ }^{6}$, D. Fugazza $^{7}$, P. Giommi ${ }^{10}$, P. Goldoni ${ }^{11}$, G. L. Israel ${ }^{6}$, \\ E. Molinari ${ }^{7}$, A. Moretti ${ }^{7}$, L. Piro ${ }^{12}$, P. Saracco ${ }^{7}$, L. Stella ${ }^{6}$, G. Tagliaferri ${ }^{7}$, and M. Vietri ${ }^{13}$ \\ ${ }^{1}$ Laboratoire AIM (UMR 7158), Service d'Astrophysique, CEA Saclay, L’Orme des Merisiers, Bât. 709, 91191 Gif-sur-Yvette, \\ France \\ 2 Instituto de Astronomía y Física del Espacio (CONICET/UBA) C.C. 67, Suc. 28, 1428 Buenos Aires, Argentina \\ e-mail: pellizza@iafe.uba.ar \\ 3 Steward Observatory, University of Arizona, 933 North Cherry Avenue, Tucson, AZ 85721, USA \\ ${ }^{4}$ Chercheur Associé, Observatoire de Paris, 75014 Paris, France \\ 5 European Southern Observatory, Alonso de Córdova 3107, Santiago 19, Chile (on leave from CEA Saclay, France) \\ 6 INAF, Osservatorio Astronomico di Roma, via di Frascati 33, 00040 Monteporzio Catone (Roma), Italy \\ 7 INAF, Osservatorio Astronomico di Brera, via E. Bianchi 46, 23807 Merate (LC), Italy \\ 8 Università degli studi di Milano-Bicocca, Dipartimento di Fisica, piazza delle Scienze 3, 20126 Milano, Italy \\ 9 INAF, Osservatorio Astrofisico di Arcetri, largo E. Fermi 5, 50125 Firenze, Italy \\ 10 Agenzia Spaziale Italiana, Science Data Center, via di Villa Grazioli, 00198 Roma, Italy \\ 11 Laboratoire Astroparticule et Cosmologie, UMR 7164, 11 Place Marcelin Berthelot, 75231 Paris Cedex 05, France \\ 12 INAF-IASF, Sezione di Roma, via Fosso del Cavaliere 100, 00133 Roma, Italy \\ 13 Scuola Normale Superiore, piazza dei Cavalieri 7, 56126 Pisa, Italy
}

Received 11 July 2006 / Accepted 30 August 2006

\section{ABSTRACT}

\begin{abstract}
Aims. We aim at detecting and determining the properties of the host galaxy of the dark GRB 050223 .
Methods. We use VLT optical/NIR images coupled to Swift X-ray positioning, and optical spectra of the host galaxy to measure its properties.

Results. We find a single galaxy within the Swift error box of GRB 050223. It is located at $z=0.584$ and its luminosity is $L \sim 0.4 L^{*}$. Emission lines in the galaxy spectrum imply an intrinsic $S F R>7 M_{\odot} \mathrm{yr}^{-1}$, and a large extinction $A_{\mathrm{V}}>2$ mag within it. We also detect absorption lines, which reveal an underlying stellar population with an age between $40 \mathrm{Myr}$ and $1.5 \mathrm{Gyr}$.

Conclusions. The identification of a host galaxy with atypical properties using only the X-ray transient suggests that a bias may be present in the former sample of host galaxies. Dust obscuration together with intrinsic faintness are the most probable causes for the darkness of this burst.
\end{abstract}

Key words. gamma rays: bursts - galaxies: starburst

\section{Introduction}

Long gamma-ray bursts (LGRBs) are brief pulses of $\gamma$-ray radiation occuring about once per day at random positions in the sky, and lasting more than $2 \mathrm{~s}$. LGRBs are accompanied by fading afterglows detected at longer wavelengths, which can be observed for months or even years. Optical and NIR afterglows are usually observed, except for a subpopulation of dark LGRBs (DGRBs). This can be attributed, in some cases, to the lack of early observations reaching faint magnitudes. In other cases deep and prompt follow-ups indicate that true DGRBs indeed exist, and that they comprise a fraction of $\sim 10 \%$ of all bursts (Jakobsson et al. 2005).

The lack of optical afterglows in DGRBs could be explained in different ways. DGRB afterglows could be intrinsically underluminous, or extincted by intergalactic Ly- $\alpha$ absorbers (if located at high redshift) or by the dusty interstellar medium of their host galaxies (HGs). While some authors find that the intrinsic

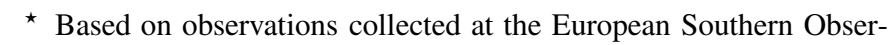
vatory, Chile, under proposal ESO 75.A-0718. faintness hypothesis could explain some cases (e.g., Pedersen et al. 2006), for other DGRBs the existence of at least some obscuration inside the HG has been claimed (e.g., Piro et al. 2002). A particularly interesting case is GRB 970828 (Groot et al. 1998; Djorgovski et al. 2001), which has been proposed as the prototype of dust-enshrouded bursts, and is the only DGRB whose HG has been detected in the FIR by Spitzer (Le Floc'h et al. 2006).

The hypothesis of dust extinction of DGRB afterglows is particularly interesting in the context of the relationship between LGRBs and star formation (SF). The connection of LGRBs with core-collapse supernovae (Hjorth et al. 2003; Stanek et al. 2003; Malesani et al. 2004; Campana et al. 2006), the SF activity observed in their HGs (Le Floc'h et al. 2002; Prochaska et al. 2004), and their massive stellar progenitors proposed by theoretical models (Fryer et al. 1999), suggest that LGRBs might be good SF tracers. Their detectability at high redshift (Tagliaferri et al. 2005; Haislip et al. 2006; Kawai et al. 2006) would then transform them in a promising tool to probe SF in the early Universe. However, the sample of HGs obtained so far shows 
a bias to underluminous and blue objects (Le Floc'h et al. 2003), while there is evidence that a significant fraction of the SF at high redshift took place in luminous, reddened and dustenshrouded IR starbursts (Elbaz et al. 2002). If the obscuration hypothesis is correct, a natural explanation for this bias arises from the fact that, in most previous investigations, LGRB HGs have been identified through the accurate positioning given by optical afterglow measurements. Hence, mostly HGs of unobscured bursts have been identified, leading to a natural exclusion of dusty starburst galaxies. The detection of such galaxies associated to LGRBs would make a strong case for the obscuration hypothesis, and would also support the LGRB-SF connection.

GRB 050223 was discovered by the Swift satellite at 03:09:06 UT on February 23, 2005 (Mitani et al. 2005), and also detected by INTEGRAL (Mereghetti et al. 2005). It was a LGRB $\left(T_{90}=23 \mathrm{~s}\right)$, with a fluence of $7.4 \times 10^{-7} \mathrm{erg} \mathrm{cm}^{-2}$ (15-350 keV). The Swift X-ray telescope (XRT) detected its afterglow (Giommi et al. 2005), which was also observed by XMM-Newton (De Luca \& Campana 2005). The afterglow presented a power-law decay with a slope $\alpha=0.99_{-0.12}^{+0.15}$. Its spectrum was well fitted by an absorbed power law with a photon index $\Gamma=1.75_{-0.18}^{+0.19}$, and a hydrogen density column $N_{\mathrm{H}} \sim$ $10^{21} \mathrm{~cm}^{-2}$, consistent with the Galactic value, $N_{\mathrm{H}}=7 \times$ $10^{20} \mathrm{~cm}^{-2}$ (Page et al. 2005). XRT gave also a precise positioning of the afterglow, $\alpha_{\mathrm{J} 2000}=18^{\mathrm{h}} 05^{\mathrm{m}} 33^{\mathrm{s}} .08, \delta_{\mathrm{J} 2000}=$ $-62^{\circ} 28^{\prime} 20^{\prime} .5$ (5'. 4 error radius; Moretti et al. 2006).

Searches for the ultraviolet and optical afterglow were made by Swift Ultraviolet and Optical Telescope (Gronwall et al. 2005), XMM Optical Monitor (Blustin et al. 2005), ROTSE III (Smith 2005), PROMPT (Nysewander et al. 2005) and the Mount John University Observatory (Gorosabel et al. 2005). No observations were reported in the IR or radio domains. The deepest observations put a stringent limiting magnitude $R>21.2$ only $4.1 \mathrm{~h}$ after the burst. Assuming that the decay of the optical afterglow follows the same law as the X-ray afterglow, and taking into account the Galactic absorption of 0.2 mag at the position of the burst (Schlegel et al. 1998), this limit implies a magnitude $R>23.8$ at 2 days after the burst. Hence, following the criterion of Djorgovski et al. (2001), GRB 050223 can be classified as a DGRB. The aforementioned observations put also an upper limit $\beta_{\mathrm{OX}}<0.8$ for the mean optical to X-ray spectral index of GRB 050223, hence it can not be classified as dark according to the criterion of Jakobsson et al. (2004), which requires $\beta_{\mathrm{OX}}<0.5$ for a burst to be dark. This is a direct consequence of the faintness of the X-ray afterglow, which was used by Page et al. (2005), together with the value of $N_{\mathrm{H}}$, to argue that GRB 050223 has an intrinsically underluminous afterglow.

In this Letter we report the detection of the host galaxy of GRB 050223 and the determination of its properties. Sections 2 and 3 present our observations and results respectively. Section 4 discusses the properties of the galaxy and their implications on the nature of DGRBs.

\section{Observations}

Our observations of GRB 050223 were carried out with the ESO 8-m Very Large Telescope UTs 1 and 2 at Paranal Observatory, Chile, as part of a program dedicated to the study of LGRB HGs (ESO 75.A-0718, P. I. Pellizza). Optical and NIR images of the field of the source were obtained with the ESO Focal Reducer and Low Dispersion Spectrograph 1 (FORS 1) on May 10, 2005, and with the Infrared Spectrometer And Array Camera (ISAAC) on June 5, 2005. FORS 1 was used in imaging mode with the Bessel $R$ filter to obtain 10 deep
(308 s per exposure) frames of the field of the source. A set of $421 \mathrm{~min}$ exposures were also taken in the short wave mode of ISAAC with the $K_{\mathrm{s}}$ filter. These images were reduced in the standard way (bias/dark subtraction, flat fielding and illumination correction), and combined to obtain very deep final science images with equivalent exposures of 51 and $42 \mathrm{~min}$ in the $R$ and $K_{\mathrm{s}}$ bands, respectively.

By chance, we found a single $\mathrm{HG}$ candidate within GRB 050223 Swift error box, down to the sensitivity limits of our data. For this candidate, we obtained optical spectra with FORS 2 on July 30-31, 2005. Three $1 \mathrm{~h}$ exposures were made in LSS mode with an 1" slit, grism 300I and the OG590 order sorting filter. Each of them was reduced (bias subtraction, flat fielding), and the three frames were combined into a single one with an equivalent exposure of $3 \mathrm{~h}$, from which the spectrum was extracted and calibrated in wavelength and flux. All reduction and calibration tasks were performed with IRAF.

\section{Data analysis and results}

We performed the astrometry of our science $R$ and $K_{\mathrm{s}}$ images using 2MASS coordinates of 22 detected stars. We obtained an rms error of 0.13 and 0.14 respectively, small enough for our purposes. In Fig. 1 we show the field of the source in both bands, together with the position of the Swift and XMM error circles (5'. 4 and $3{ }^{\prime \prime} 1$ radius at $90 \%$ CL respectively), the latter obtained from our own analysis of XMM data. By chance, we find a single object (H in Fig. 1) inside both circles. Three other objects ( $\mathrm{S}$ in Fig. 1) lie outside them but near their boundaries; they are stars with $>99 \%$ probability according to a PSF fit to their profiles. Object $\mathrm{H}$ is extended (roughly $11^{\prime \prime} 5$ in radius) and morphologically consistent with a galaxy. Its coordinates (J2000) are $\alpha=18^{\mathrm{h}} 05^{\mathrm{m}} 32^{\mathrm{s}} .992, \delta=-62^{\circ} 28^{\prime} 18^{\prime \prime} .81\left( \pm 0^{\prime}{ }^{\prime} 16\right.$, which takes into account the 2MASS catalog uncertainty of 0.1 ), only 1. .'8 apart from the nominal XRT afterglow position, and 2".7 from that of $X M M$. There is no other conspicuous object within the error circles down to our $3 \sigma$ limiting magnitudes of $R \sim 26$ and $K_{\mathrm{s}} \sim 21$. We note that with these limits a typical LGRB HG like those in the sample of Le Floc'h et al. (2003) would be detectable up to a redshift of $\sim 2.5$. Hence, although at this point we cannot completely discard a high redshift galaxy, $\mathrm{H}$ is a good $\mathrm{HG}$ candidate for GRB 050223 .

We performed differential aperture photometry of candidate H, using a set of stars in our frames with known USNO B1.0 and 2MASS as comparisons. $R$ magnitudes of the comparison stars were computed from their photographic IIIa-J and IIIa-F magnitudes and the transformations of Windhorst et al. (1991), while their $K_{\mathrm{s}}$ magnitudes were taken directly from the 2MASS catalog. Linear fits of comparison against instrumental magnitudes give $R=21.76 \pm 0.05$ and $K_{\mathrm{s}}=18.88 \pm 0.02$ for candidate $\mathrm{H}$. Correcting for Galactic extinction $(E(B-V)=$ 0.09; Schlegel et al. 1998), we obtain its intrinsic magnitudes, $R=21.55 \pm 0.05$ and $K_{\mathrm{S}}=18.85 \pm 0.02$. Using galaxy number counts (Liske et al. 2003), we derive probabilities of $\sim 5 \%$ and $\sim 2 \%$ of finding a galaxy brighter than candidate $\mathrm{H}$ within the XRT and XMM error circles, respectively. Computing the fraction of the sky area covered by galaxies brighter than $\mathrm{H}$ (e.g., Piro et al. 2002) gives a probability of chance alignment of $1 \%$, consistent with the previous values. Hence, we can discard at $>95 \% \mathrm{CL}$ the hypothesis that $\mathrm{H}$ is a field galaxy aligned by chance with the burst, making a stronger case for its association to GRB 050223 .

In Fig. 2 we show our spectrum of candidate $\mathrm{H}$; the features identified in it are listed in Table 1. The spectrum presents 

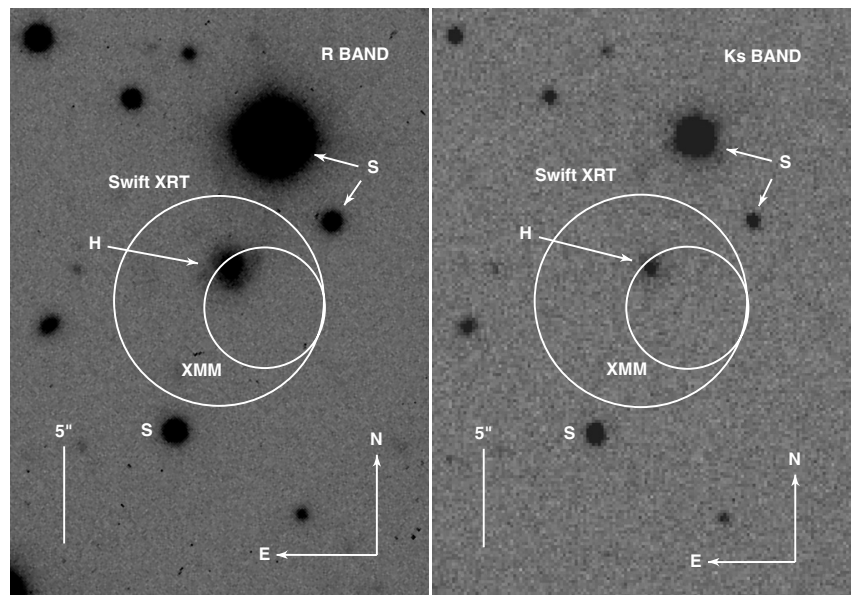

Fig. 1. The field of GRB 050223 in $R$ (left panel) and $K_{\mathrm{S}}$ (right panel) bands. North is up and East is to the left. The large and small circles are the Swift XRT and XMM error boxes for the source (5". 4 and 3". 1 radius respectively at $90 \% \mathrm{CL})$. A single extended object $(\mathrm{H})$ is found inside them in both images. Other three objects $(\mathrm{S})$ lying near their boundaries are stars with $>99 \%$ probability.
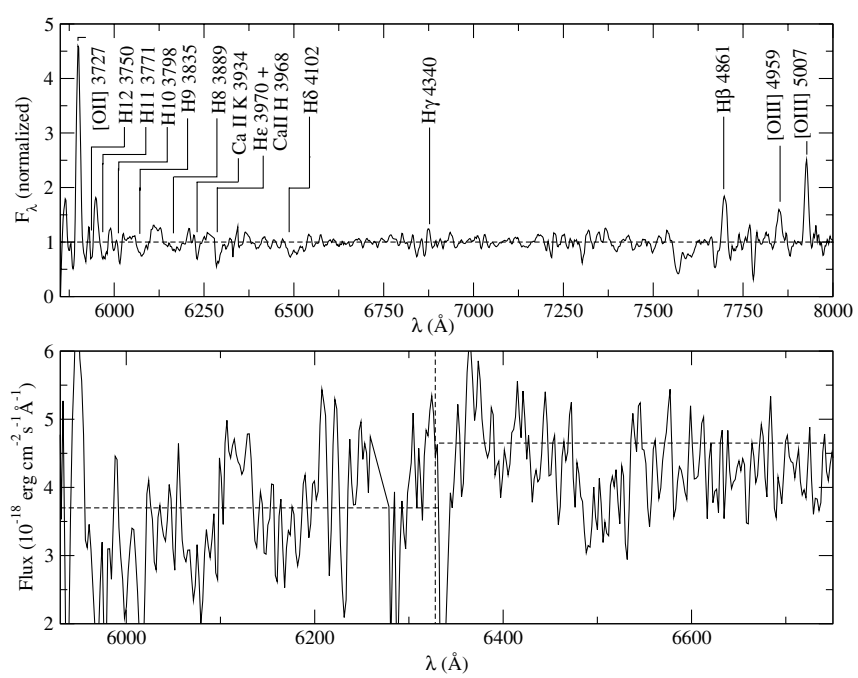

Fig. 2. Upper panel: normalized optical spectrum of candidate $\mathrm{H}$, showing lines both in emission $(\mathrm{H} \beta, \mathrm{H} \gamma,[\mathrm{O} \mathrm{II}],[\mathrm{O} \mathrm{III}])$ and absorption $(\mathrm{H} \delta$, Ca II, early Balmer lines). Lower panel: flux-calibrated spectrum of the region around $4000 \AA$ (rest frame, vertical dashed line), exhibiting the break at this wavelength. Horizontal dashed lines are plotted at the mean flux in the 3750-3950 ̊ and 4050-4250 ̊ ranges.

prominent emission lines (H $\beta, \mathrm{H} \gamma$, [O II] $3727 \AA$, [O III] 4959, $5007 \AA$ ). Although the $\mathrm{S} / \mathrm{N}$ ratio is poor, we identify also a rather strong stellar continuum exhibiting a clear break at $4000 \AA$ (rest frame), and absorption lines such as strong $\mathrm{H} \delta$, Ca II lines, and several early Balmer lines. From the emission lines we derive a redshift $z=0.584 \pm 0.005$, consistent with that obtained by Berger \& Shin (2006). At this redshift, the luminosity distance of the galaxy (for a cosmological model with $\Omega_{\Lambda}=0.7, \Omega_{\mathrm{m}}=0.3$ and $H_{0}=70 \mathrm{~km} \mathrm{~s}^{-1} \mathrm{Mpc}^{-1}$ ) is $3.4 \mathrm{Gpc}$. Using this distance and the k-correction for an Sc model (Poggianti 1997), we derive an absolute magnitude $M_{K_{\mathrm{s}}}=-23.47 \pm 0.03$. Assuming $M_{K_{\mathrm{s}}}^{*}=-24.5$ (e.g., Saracco et al. 2006) the luminosity of the galaxy is $L \sim 0.4 L^{*}$, which makes $\mathrm{H}$ one of the most luminous LGRB HGs. The blue $R-K_{\mathrm{s}}=2.70 \pm 0.07$, though not unusual,
Table 1. Lines identified in the spectrum of candidate $\mathrm{H}$.

\begin{tabular}{lccc}
\hline \hline Species & $\begin{array}{c}\lambda_{\text {lab }} \\
(\AA)\end{array}$ & $\begin{array}{c}\lambda_{\text {obs }} \\
( \pm 1 \AA)\end{array}$ & $\begin{array}{c}E W \text { (rest frame) } \\
(\AA)\end{array}$ \\
\hline$[$ O II] & 3727 & 5900 & $-27 \pm 6$ \\
H12 & 3750.154 & 5937 & $2.3 \pm 0.3$ \\
H11 & 3770.632 & 5972 & - \\
H10 & 3797.900 & 6015 & $2.6 \pm 0.6$ \\
H9 & 3835.386 & 6077 & - \\
H8 & 3889.051 & 6167 & - \\
Ca II K & 3933.664 & 6232 & $<2.6$ \\
Ca II H & 3968.470 & 6285 & - \\
H $\varepsilon$ & 3970.074 & 6289 & - \\
H $\delta$ & 4101.737 & 6496 & $4.4 \pm 0.6$ \\
H $\gamma$ & 4340.468 & 6875 & $>-2.4$ \\
H $\beta$ & 4861.332 & 7699 & $-8.2 \pm 2.0$ \\
{$\left[\mathrm{O}_{\text {III }}\right]$} & 4959 & 7850 & $-5.7 \pm 1.8$ \\
{$[$ O III $]$} & 5007 & 7927 & $-11.3 \pm 1.3$ \\
\hline
\end{tabular}

is redder than those of LGRB HGs at $z \sim 0.6$ (Le Floc'h et al. 2003).

The detection of $\mathrm{H} \delta$ and early Balmer lines in absorption, while $\mathrm{H} \gamma$ and $\mathrm{H} \beta$ are seen in emission, implies a strong Balmer decrement and suggests a large extinction. Assuming $R_{V}=3.1$, the ratio $\mathrm{H} \gamma / \mathrm{H} \beta<0.3$ (corrected for Galactic extinction) gives $A_{\mathrm{V}}>2 \mathrm{mag}$, or $E(B-V)>0.65$ within $\mathrm{H}$. This corresponds to a hydrogen column density $N_{\mathrm{H}} \gtrsim 10^{21} \mathrm{~cm}^{-2}$ (Dickey \& Lockman 1990), marginally consistent with that derived from X-ray observations (Page et al. 2005). The observed emission lines indicate the presence of ongoing star formation. We measure a lower limit ${ }^{1}$ to the [O II] $3727 \AA$ flux $F_{[\text {OII] }}>$ $8.2 \times 10^{-17} \mathrm{erg} \mathrm{cm}^{-2} \mathrm{~s}^{-1}$, corrected for Galactic extinction, which corresponds to a luminosity $L_{[\mathrm{OII}]}>1.3 \times 10^{41} \mathrm{erg} \mathrm{s}^{-1}$. Correcting for the measured extinction within $\mathrm{H}$, we derive a $S F R>7 M_{\odot} \mathrm{yr}^{-1}$ (Kennicutt 1998), and hence a specific $S F R>$ $17.5 M_{\odot} \mathrm{yr}^{-1} L^{*-1}$. We do not detect the [Ne III] $3869 \AA$ A line, implying a low $[\mathrm{Ne} \mathrm{III}] /[\mathrm{O} \mathrm{II}]$ ratio.

The rest-frame equivalent widths of $\mathrm{H} \delta$ and [O II] $3727 \AA$ are consistent with an e(a) classification for this galaxy $\mathrm{e}(\mathrm{a})$ galaxies are emission-line objects with strong $\mathrm{H} \delta$ absorption, $E W(\mathrm{H} \delta)>4 \AA$, and moderate [O II] emission, $5 \AA<\mid E W(\mathrm{O}$ II $) \mid<40 \AA$ (Poggianti \& Wu 2000) -. This is also consistent with the derived extinction, as e(a) galaxies have a median $E(B-V)=1.11$. The probability of finding an e(a) galaxy within the XRT error circle is less than $0.5 \%$ (Poggianti \& Wu 2000), making a stronger case for the association between this galaxy and GRB 050223. The presence of a strong $\mathrm{H} \delta$ line and several early Balmer lines $(\mathrm{H} \varepsilon-\mathrm{H} 12)$ in absorption indicates also the existence of a stellar population dominated by $\mathrm{A} 0 \mathrm{~V}$ stars, whose age could range from $40 \mathrm{Myr}$ to 1.5 Gyr (Poggianti \& Wu 2000). Finally, the observed $4000 \AA$ break has an index $D_{4000}=1.3 \pm 0.2$, which is consistent with late-type star-forming galaxies.

\section{Discussion}

Combining the accurate X-ray positioning of LGRB afterglows given by Swift XRT, and very deep VLT optical and NIR images and optical spectra, we identified the likely HG of the dark GRB 050223. It is a $0.4 L^{*}$, blue galaxy at $z=0.584$, with

1 The galaxy is larger than the slit width, but we expect this limit to be near the true value because only the faint outskirts were missed. 
strong SF $\left(S F R>7 M_{\odot} \mathrm{yr}^{-1}, \operatorname{SSFR}>17.5 M_{\odot} \mathrm{yr}^{-1} L^{*-1}\right)$, a stellar population dominated by A0V stars with an age of $40 \mathrm{Myr}-1.5 \mathrm{Gyr}$, and a large extinction $\left(A_{\mathrm{V}}>2 \mathrm{mag}\right)$.

This galaxy has some typical properties of LGRB HGs. It is underluminous and blue, with strong emission lines indicating SF, although it is brighter and redder than other LGRB HGs at its redshift (Le Floc'h et al. 2003). It shows also some atypical features. The [Ne III] $3869 \AA$ to [O II] $3727 \AA$ ratio is low, and the inferred SFR and extinction are among the highest ones derived from optical observations (much higher SFRs were determined from radio observations, e.g. by Berger et al. (2003), although radio SFRs are in some cases higher than optical ones for the same galaxy). Another atypical property is the presence of a strong stellar population dominated by A0V stars, implied by the observed absorption lines, and the e(a) classification that follows from them. We point out that our finding of an HG spectrum that differs from those previously reported is the result of a new HG selection based only on X-ray transients. This may imply the presence of a bias in the HG sample that has been considered so far.

The large observed extinction suggests that this DGRB took place in a dust-enshrouded starburst. The e(a) type spectrum is usually interpreted as produced in galaxies with strong ongoing star formation, together with older stellar populations and a high selective obscuration affecting the youngest stars (Poggianti et al. 2001). Star forming regions, inside which LGRBs occur, are expected to be highly obscured in these galaxies. Hence, our results support the obscuration hypothesis for DGRBs, which was also strengthened by the recent classification of the HG of GRB 030115 as an extremely red object (Levan et al. 2006). Our picture is consistent with that of Nakagawa et al. (2006), who proposed that the dark GRB 051022 took place inside a dusty molecular cloud, given the large extinction $\left(A_{\mathrm{V}}=49 \mathrm{mag}\right) \mathrm{de}-$ rived from X-ray data. We point out that a very high dust extinction has been also proposed as a possible reason for the discrepancy between the rate of core-collapse $\mathrm{SNe}$ (which are related to LGRBs) determined from NIR surveys, and that estimated from the FIR luminosity of their parent galaxies (Mannucci et al. 2003). However, we note that the only LGRB with a HG possibly showing a similar extinction $\left(A_{\mathrm{V}}=3.4 \mathrm{mag}\right.$ according to Vreeswijk et al. 2001, but only 0.15 mag according to Christensen et al. 2004) to that of our candidate, is not a DGRB (GRB 990712 at $z=0.43$ ). If its high extinction is confirmed, this could be the result of a different dust distribution in both galaxies, obscuration not affecting the star-forming regions in the HG of GRB 990712 (not an e(a) type). Or, this might imply that dust extinction alone is not a sufficient condition to produce a DGRB. On the other hand, the confirmation of a low extinction for the GRB 990712 HG would strengthen the hypothesis that dusty strabursts produce DGRBs. It is important to note, however, that there are DGRB HGs which are not representative of dusty starbursts (e.g., GRB 000210; Gorosabel et al. 2003).

Our measurement of a redshift $z=0.584$ for the likely host galaxy of GRB 050223 casts a shadow over the high redshift hypothesis proposed by Page et al. (2005) to explain the faintness of its X-ray afterglow, leaving the possibility of a large jet opening or viewing angle. Our detection of obscured star formation in its HG does not contradict their conclusion of the GRB 050223 afterglow being underluminous. Intrinsic faintness might well be complementary to dust absorption in producing a DGRB, either by requiring a modest dust mass to suppress the afterglow, or by being ineffective in destroying the circumburst dust. We note that GRB 050223 and GRB 990712 lie at opposite ends in the $\mathrm{X}$-ray afterglow brightness distribution.

Although it shows a high optical extinction, the likely HG of GRB 050223 does not seem to be one of the galaxies with luminous, reddened IR starbursts and very high SF activity. Particularly, it was not detected by Spitzer (Le Floc'h 2006, priv. comm.) in the FIR. The constraint set by these observations, $S F R<10 M_{\odot} \mathrm{yr}^{-1}$, is consistent with our results. However, it is noteworthy that the position of this HG in Figs. 4 and 5 of Le Floc'h et al. (2003) is consistent with the loci of the NIR counterparts of high redshift ISO galaxies. Further investigation of this $\mathrm{HG}$ would be important to get a deeper insight into the nature of DGRBs and the LGRB-SF connection.

Acknowledgements. We acknowledge D. Malesani for a careful reading of the manuscript and useful comments on it, and the anonymous referee for suggestions that greatly improved this work. The MISTICI activities in Italy are supported by ASI grant I/R/039/04. This publication makes use of data products from the Two Micron All Sky Survey, which is a joint project of the University of Massachusetts and the Infrared Processing and Analysis Center/California Institute of Technology, funded by the National Aeronautics and Space Administration and the National Science Foundation.

\section{References}

Berger, E., \& Shin, M.-S. 2006, GCN, 5283

Berger, E., Cowie, L. L., Kulkarni, S. R., et al. 2003, ApJ, 588, 99 Blustin, A., Branduardi-Raymont, G., Breeveld, A., et al. 2005, GCN, 3093 Campana, S., Mangano, V., Blustin, A. J., et al. 2006, Nature, 442, 1008 Christensen, L., Hjorth, J., Gorosabel, J., et al. 2004, A\&A, 413, 121 De Luca, A., \& Campana, S. 2005, GCN, 3109

Dickey, J. M., \& Lockman, F. J. 1990, ARA\&A, 28, 215

Djorgovski, S. G., Frail, D. A., Kulkarni, S., et al. 2001, ApJ, 562, 654

Elbaz, D., Cesarsky, C. J., Chanial, P., et al. 2002, A\&A, 384, 848

Fryer, Ch., Woosley, S. E., \& Hartmann, D. H. 1999, A\&A, 526, 152 Giommi, P., Capalbi, M., Perri, M., et al. 2005, GCN, 3054

Gorosabel, J., Christensen, L., Hjorth, J., et al. 2003, A\&A, 400, 127 Gorosabel, J., Eguchi, S., de Ugarte Postigo, A., et al. 2005, GCN, 3061 Gronwall, C., Blustin, A., Brown, P., et al. 2005, GCN, 3057

Groot, P. J., Galama, T. J., van Paradijs, J., et al. 1998, ApJ, 493, L27 Haislip, J. B., Nysewander, M. C., Reichart, D. E., et al. 2006, Nature, 440, 181 Hjorth, J., Sollerman, J., Moller, P., et al. 2003, Nature, 423, 847 Jakobsson, P., Hjorth, J., Fynbo, J. P. U., et al. 2004, ApJ, 617, L21 Jakobsson, P., Frail, D. A., Fox, D. B., et al. 2005, ApJ, 629, 45

Kawai, N., Kosugi, G., Aoki, K., et al. 2006, Nature, 440, 184 Kennicutt, R. J. 1998, ARA\&A, 36, 189

Le Floc'h, E., Duc, P.-A., Mirabel, I. F., et al. 2002, ApJ, 581, L81 Le Floc'h, E., Duc, P.-A., Mirabel, I. F., et al. 2003, A\&A, 400, 499 Le Floc'h, E., Charmandaris, V., Forrest, W., et al. 2006, ApJ, 642, 636 Levan, A., Fruchter, A., Rhoads, J., et al. 2006, ApJ, 647, 471 Liske, J., Lemon, D. J., Driver, S. P., et al. 2003, MNRAS, 344, 307. Mereghetti, S., Gotz, D., Mowlavi, N., et al. 2005, GCN, 3059 Malesani, D., Tagliaferri, G., Chincarini, G., et al. 2004, ApJ, 609, L5 Manucci, F., Maiolino, R., Cresci, G., et al. 2003, A\&A, 401, 51 Mitani, T., Barbier, L., Barthelmy, S., et al. 2005, GCN, 3055 Moretti, A., Perri, M., Capalbi, M., et al. 2006, A\&A, 448, L9 Nakagawa, Y. E., Yoshida, A., Sugita, S., et al. 2006, PASJ, 58, L35 Nysewander, M., Bayliss, M., Haislip, J., et al. 2005, GCN, 3067 Page, K. L., Rol, E., Levan, A. J., et al. 2005, MNRAS, 363, L76 Pedersen, K., Hurley, K., Hjorth, J., et al. 2006, ApJ, 636, 381 Piro, L., Frail, D. A., Gorosabel, J., et al. 2002, ApJ, 577, 680 Poggianti, B. M. 1997, A\&AS, 122, 399

Poggianti, B. M., \& Wu, H. 2000, ApJ, 529, 157

Poggianti, B. M., Bressan, A., \& Franceschini, A. 2001, ApJ, 550, 195 Prochaska, J. X., Bloom, J. S., Chen, H.-W., et al. 2004, ApJ, 611, 200 Saracco, P., Fiano, A., Chincarini, G., et al. 2006, MNRAS, 367, 349 Schlegel, D. J., Finkbeiner, D. P., \& Davis, M. 1998, ApJ, 500, 525 Smith, D. A. 2005, GCN, 3056

Stanek, K. Z., Matheson, T., Garnavich, P. M., et al. 2003, ApJ, 591, L17 Tagliaferri, G., Antonelli, L., Chincarini, G., et al. 2005, ApJ, 443, L1 Vreeswijk, P. M., Fruchter, A., Kaper, L., et. al. 2001, ApJ, 546, 672 Windhorst, R., Burstein, D., Mathis, D. F., et. al. 1991, ApJ, 380, 362 